\title{
Data Exchange Service using Google Drive API
}

\author{
Risky Cahya Dinatha \\ Department of Information \\ Technology, Udayana \\ University, Bali, Indonesia
}

\author{
I. Made Sukarsa \\ Department of Information \\ Technology, Udayana \\ University, Bali, Indonesia
}

\author{
A. A. K. Agung \\ Cahyawan \\ Department of Information \\ Technology, Udayana \\ University, Bali, Indonesia
}

\begin{abstract}
Data Exchange Service Using Application Programming Interface (API) Google Drive is a web based service that can exchange data through the Internet. This service utilizes Google Drive cloud storage as an intermediary and data storage media. Google Drive as a solution to the problem of interoperability is the ability of the system to take advantage of the information being exchanged. The purpose of this service is to help the business processes of an organization and provides an alternative communication way if a problem occurs in the main system. One of the advantages of the method of data exchange by utilizing the Google Drive cloud storage is not bound by the Internet Protocol address so that the operation can be done anywhere just with the Internet network. This service requires a Google account that is used for authentication and as the sender's identity data.
\end{abstract}

\section{Keywords}

Data Exchange Service, Google Drive, API, Google Drive REST API, Cloud Storage

\section{INTRODUCTION}

Information has become a fundamental importance in human life. Information obtained from the communication between several individuals or groups. Internet technology combined with a computer system can realize long-distance communication at the same time making it easier for certain people to get the desired information.

Data exchange is the process of moving data from a computer system to another computer system. The computer system can receive data from or send data to another computer systems and applies vice versa. Good data exchange will occur when data is exchanged then can be received and understood by the computer systems. The challenges in data exchange is the ability to understand the file format and the interaction between computing resource or application in the data processing that can be done through various communication channels.

Data Exchange Service Using Google Drive API is a webbased application in which utilize APIs from Google Drive and incorporate a wide variety of programming languages into one file so that it becomes a web service engine. This concept is used not only to solve problems but also facilitate interoperability in terms of connectivity as it can be used anywhere and anytime through the Internet network without the need to know the Internet Protocol address and access to a server owned by the recipient.

\section{RELATED WORKS}

This study can be attributed to the reviews, references, or the underlying source in the system design. The discussion of the underlying theory in the making of this system is described as follows.
Jamil Cahyadi et al conducted a study titled Data Exchange Architecture based-on Data Grid in Developing Gorontalo Library Network describes a system that connects many libraries in order to facilitate public access to information in the area in every library that is connected through this system [1]. Gorontalo Library Network (GLN) connects every local library that has formed a digital library despite the differences in terms of the platform with the help of grid technology. GLN is a library in the library of the province and six counties the local library or city. The scenario of the data exchange process between the digital library is to find books in the library A, if the book is not found in the library A and library A sends a request to the server to search a collection of books in other libraries, other libraries sent a reply to the server through a collection of books that sought, and the server will send search results to a library by including the physical location of the requested books.

Cloud Storage Design with Auto Syncing Concept Using Owncloud and Dropbox Apps by Jamil Cahyadi et al which discusses synchronization Dropbox cloud storage through the Owncloud application [2]. External Storage features on the Owncloud used to set the Dropbox API into it. API which has been set will result in a folder that has been synchronized to Dropbox and Owncloud (local) so, the file that has been uploaded to the Dropbox will be synchronized to the local machine. Advantages of using this method is to suppress the use of bandwidth because synchronization can be performed on one machine and the other machines can access these files via the local network without the need to connect to the Internet.

Data Exchange Between Information System at Low Bandwidth Quality Using Messaging by I Made Sukarsa et al [3]. This study uses Yahoo Messenger to conduct two-way communication between accounts where content is exchanged as data format or text can be longer than the Short Message Services (SMS) and this way does not require a large bandwidth. The system architecture developed in this research is to build-Based Messaging Middleware (MOM) as an interpreter or translator between the existing system. MOM is designed to connect to the MOM database itself and the external database (database system). MOM database contains metadata for a business process that is used in data exchange between applications. The external database is an existing database that is used by the system. The external database is the source of the data exchange is carried out by MOM. The data exchange to other applications via the MOM that already exist in other systems so each system has its own MOM because each system has a different database and thus require different interpreter.

\section{BACKGROUND KNOWLEDGE 3.1 Cloud Storage}

Cloud Storage is a service where the data is maintained, managed and backed up. This service is available to a party 
who normally use the Internet connection. It allows the parties to store files online so you can access from any location via the Internet. The provider keeping the data on its servers so that data can be accessed online by the owner. The file owner should be aware that the data backup of the file is still required when using a cloud storage storage media, for recovering data from cloud storage is much longer than the local backup [4].

\subsection{Google Drive}

Google Drive is a cloud application provided by Google as a storage medium that can be integrated into a variety of other Google applications. Google Drive release on 24 April 2012 which initially is Google Docs. Google Docs which initially had docs.google.com url changed to drive.google.com. The size of the storage media provided by Google Drive is 15GB, including Gmail and Google Photos. Storage included in the count of Google Drive is the entire message and attachment in the spam folder and the trash, PDFs, images and video. All documents created with Google Docs doc, sheet and slide does not include to the calculation of Google Drive storage. Google Photos are included to the calculation of Google Drive storage at the time the photo was uploaded using the "Original" option. Files are shared with others is not included to the calculation of Google Drive storage [5]

\subsection{Application Programming Interface (API)}

APIs are the rules of the software program that can be used to access, utilize the services and resources provided by the program which has been implemented by the API. API function is as an interface between different software programs and facilitates their interaction is similar as facilitating the interaction between humans and computers.

\subsection{Message-Oriented Middleware (MOM)}

Middleware is designed to help manage the complexity and heterogeneity inherent in distributed systems [6]. There are several types of middleware and one of them is the MessageOriented Middleware (MOM). MOM is a middleware that enables communication through messages. MOM provides a message service between the transport layer and application layer of the network protocol stack [7].

\subsection{Web Service}

Web service is a software system designed to support interoperability and interaction between systems on a network [8]. Web service is used as a facility provided by a website to provide services in the form of information to other systems, so that other systems can interact with the system through the services (service) provided by a system that provides a web service. Web service data store information in XML format, so that this data can be accessed by other systems, although different platforms and operating systems.

\subsection{Electronic Data Interchange (EDI)}

Electronic Data Interchange (EDI) is a method of exchanging business documents between computer applications in the enterprise / agency electronically using a standard format that has been agreed between the two parties which relate that have different systems and applications associated with EDI technology [9]. EDI has three main components, namely the standard messages, EDI software, and communications. The first component contains the standard text message that includes information and rule as a translator of one or more business documents. The second component of the Software EDI serves as the interpreters of the standard EDI message to an internal file format of the receiving company. The third component in the EDI communication is done through intermachine (computer) so the communications infrastructure is required. Forms of communication infrastructure that was first developed is a transaction in the form of point-to-point, namely the direct connection of two companies that transact. In a point-to-point in the company EDI transactions require the use of the same communication protocol, have the same transmission speed, providing a phone line at the same time [10].

\subsection{Task Scheduler}

Task Scheduler is a component of the Microsoft Windows operating system that provides the ability to perform routine tasks automatically. The task you want to run must be arranged in advance at a predetermined time or after a certain time interval. Task Scheduler plays an important role in this study as one of the features in this study require scheduling to run a script to send and receive data.

\section{DEVELOPMENT MATERIAL AND METHOD}

\subsection{Development Flowchart}

Flowchart depicting the development of the design and manufacturing process system is shown in Figure 1 below.

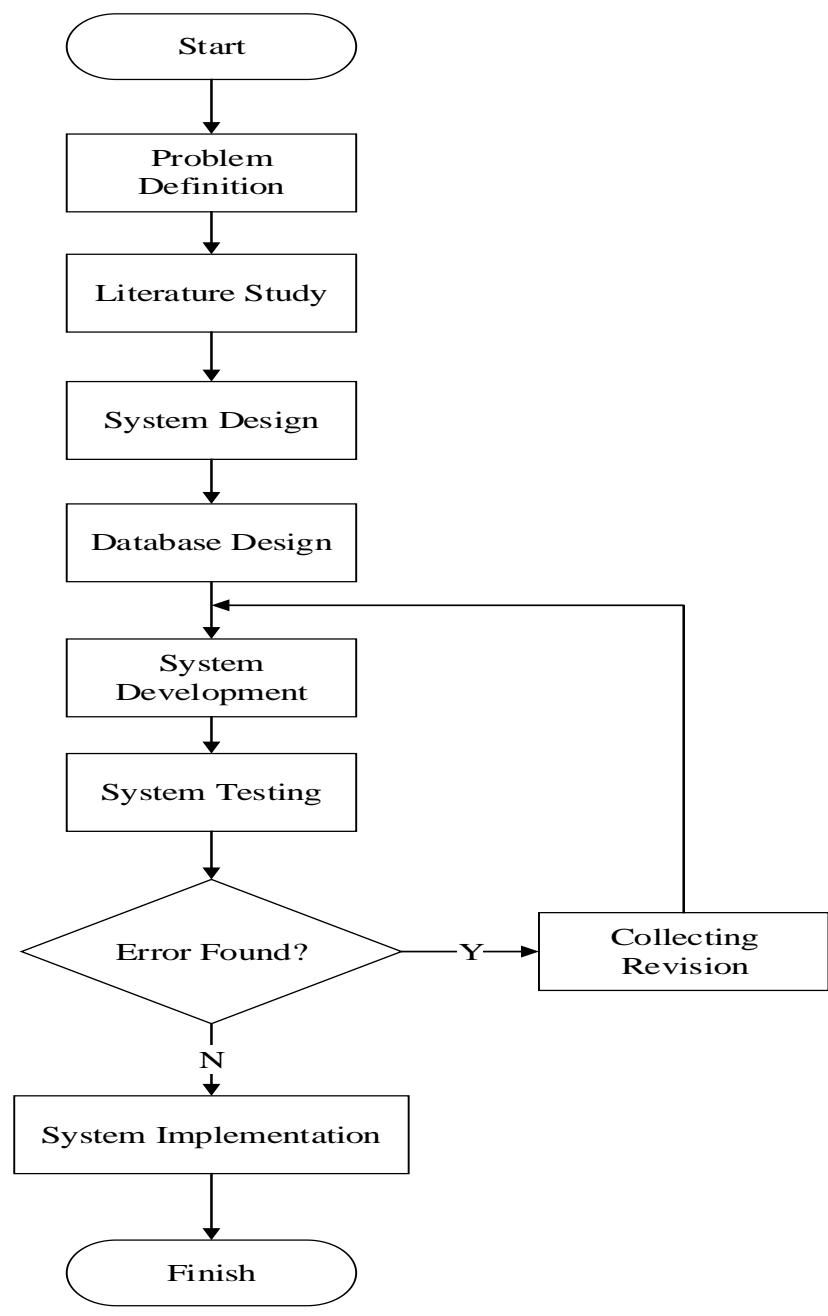

Figure 1: System Development Flowchart

The first phase is problem definition which defining the problems that found concerning the data exchange service. Second phase is literature study which collecting some data 
relating to the design and manufacture of data exchange eg examine the response data from Goolge Drive and Google+ API. Then, system design studying and understanding the processes that occur in making data exchange (utilization data formats, web services and Google Drive API) that can be made of a modeling system. Database design and manufacture structures using MySQL database. The development of data exchange service implementating web-based programming languages (HTML, PHP, and Javascript) by utilizing the Google Drive API. The system testing phase is testing the software that has been created, which, if the test fails, the revision of the system are made and then go back to the system development phase, and if the test is successful then proceed to the last phase. The last phase is implementating the system to test the data exchange between computer systems.

\subsection{System Architecture}

System architecture on the Data Exchange Service Using Google Drive API shows the interaction between the components in each layer of the system. Here is a picture of architectural system that can be seen in Figure 2.

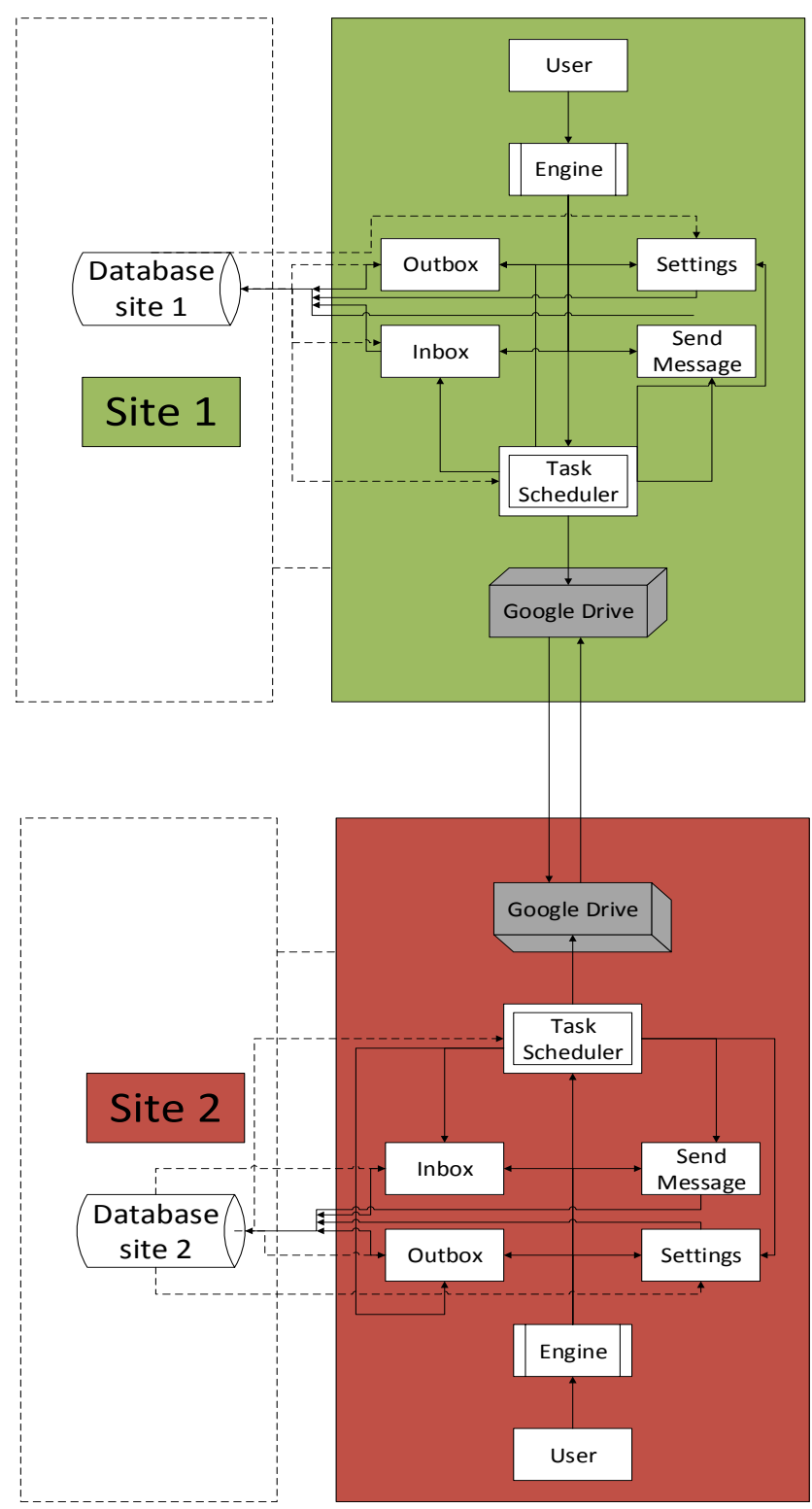

Figure 2: System Architecture
Layes in sites 1 and 2 consists of user, inbox, outbox, new messages, settings, Task Scheduler, and engine component. Engine components running inbox, outbox, new messages, settings, and Task Scheduler component according to the commands given by the user. New messages, inbox, outbox, settings, engine, and Task Scheduler to have query interaction to the database components. Inbox, outbox, and Task Scheduler receive query results from the database components that have been requested before. Google Drive components mediates the interaction between the site with other site. Relationship of a site with Google Drive components must be through the Internet network.

\section{RESULT \\ 5.1 Front Page}

The front page of the system is used as the authentication page redirects to Google. A party who has previously logged without sign out will not be redirected to the Google authentication but can be directly entered into the application.

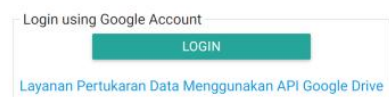

Figure 3: Front Page

Figure 3 shows the front page that displays a button to login to the system. Transitioning from the page will continue to authentication page of Google or to the main menu of the system depending on the session on the system.

\subsection{Authentication}

Transitioning from the front page of the system when certain parties have not logged in using their Google account.

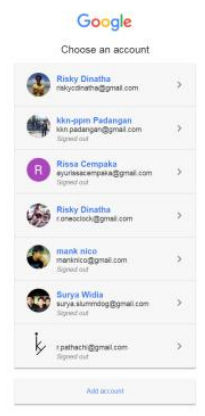

Figure 4: Choosing or Logging In with Google Account

Figure 4 shows the selection page Google account where a party may choose accounts that have provided or add a new account by clicking the add account button. Accounts that are displayed on this page is an account that has been logging stored in the browser cache. 


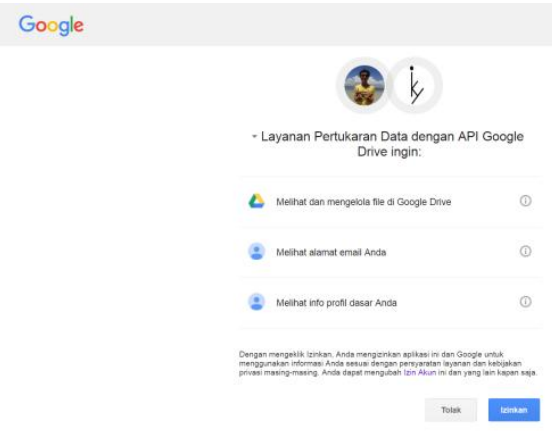

Figure 5: Authentication Page

Figure 5 shows the authentication page displaying scope of information use and management of files on Google Drive along with other information in order to be allowed to use in this application.

\subsection{Inbox}

Inbox page is part of receipt message displays the list of incoming messages. The message is received if the other account owner sending the message to the recipient's email address. Technically messaging occurs on Google Drive cloud with file upload process and share those files to the destination account.

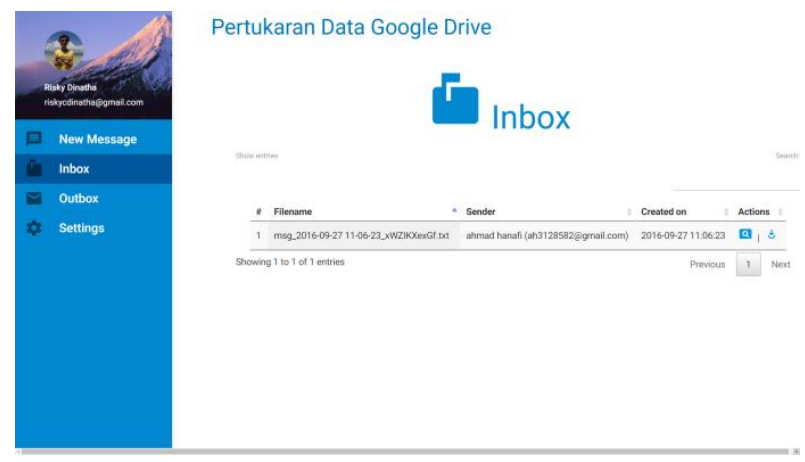

Figure 6: Inbox Page

Figure 6 shows the inbox page that displays the file name, sender, date the message was made and actions that are used to open the message. The message list can be sorted by name, sender, or date.

\subsection{New Message}

New message pages is part of the message delivery. Users who wish to send the message must use the new message page and fill out the form.

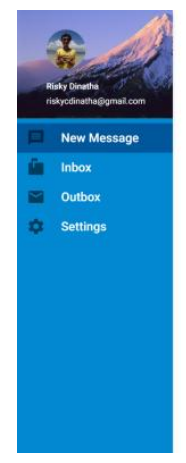

Pertukaran Data Google Drive

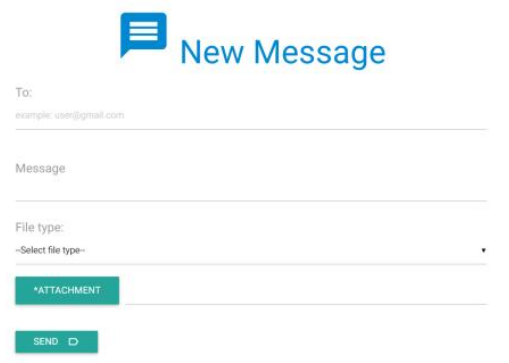

Figure 7: New Message

Figure 7 shows the new message page that displays the form for sending a message. Users must fill in the destination and file type messages while the message body and attachments are optional. The completed form will be processed by the system by clicking on the Send button and wait for a message from the system if the delivery has been a success or an error. Errors that occur in the form of delays in message delivery due to excessive request on Google Drive. Pending messages have been saved by the system and the system will try sending the message again automatically through scheduling service messages.

\subsection{Outbox}

Outbox pages is part of the message delivery. Outbox page displays a list of outgoing messages that have been sent by the user.



Figure 8: Outbox Page

Figure 8 is an outbox page that displays a list of outgoing messages in the form of a file name, destination address, the date it was made and the actions that are used to open the message. The message list can be sorted by name, destination, or date.

\subsection{View Message}

View message page is used to view information of the message that can be opened from inbox or outbox page.

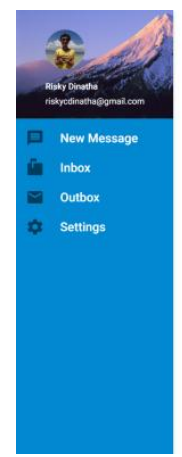

Pertukaran Data Google Drive

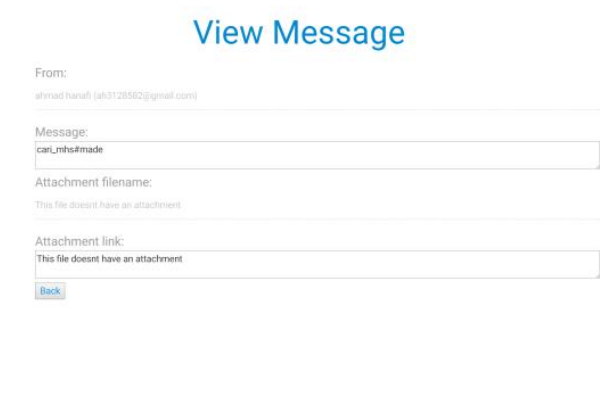

Figure 9: View Message Page

Figure 9 shows the view message page that displays the sender's address or destination address of a message, the message content, attachment file names, file attachments and links.

\subsection{Settings}

Some of the features of the system can be set via the settings page. Implementation of the system setting is performed to determine whether the settings are run in accordance with their respective functions. 




\section{कै settings}

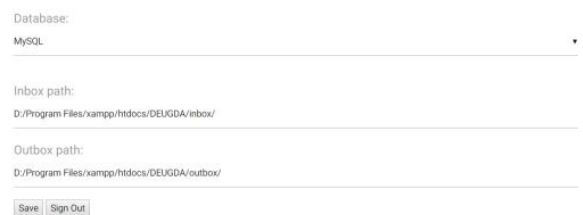

Figure 10: Settings Page

Figure 10 shows the settings page that displays a form to make settings in the system. The database is part of the form that is used to regulate the use of the database. Database settings has two options No and MySQL. No used so that the system runs without using a database while MySQL is used for the system to run using MySQL database. The suggested system runs using the database because some features can not be run without the database. Inbox and outbox path is used to set the local directory where it keeps a file of messages and attachments. Inbox path represents the directory path of incoming messages and outbox path represents the directory path of outgoing messages. Save button serves to store the data settings. The account owner who wants signout of the system or want to replace the account being able to do it by clicking sign out button and then the owner of the account will be brought to the front page.

\subsection{Message Scheduling Services}

This feature uses the Task Scheduler that runs in the background on the server side. This feature allows a party can receive and send messages without opening a web application. Sending messages can be done through the database by entering some important fields. Task Scheduler gives the command to run the most of the scripts from the engine that occurs every minute to send or receive messages.

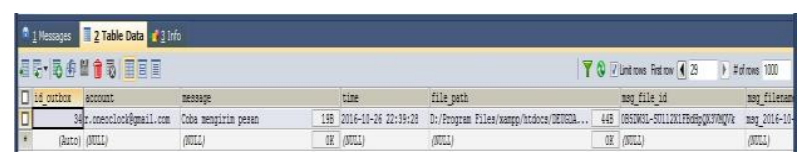

Figure 11: Outbox Page

Figure 11 shows the results of message scheduling service features. The process is to check the contents of the inbox and match tables with data in Google Drive by entering multiple search parameters file. The results of the matching process is data that does not fit so that data can be added to the database as a new message data.

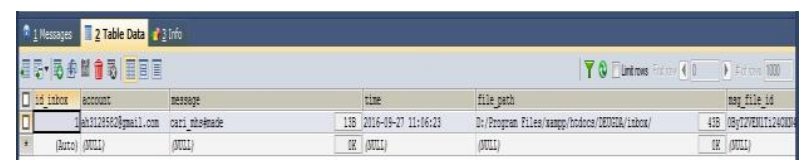

Figure 12: tb_outbox in Database

Figure 12 shows the results of message scheduling service features. The process is to check the status of the outgoing messages that 0 or delayed. Each delayed outgoing message will be resubmitted to the terms field accounts, file path, and msg_filename in tb_outbox must be filled correctly.

\section{CONCLUSION}

Data Exchange Service Using Google Drive API is done by utilizing the Google Drive cloud storage, using the Google+ API, Google Drive API, MySQL database, PHP programming language, HTML, Javascript, and Materialize CSS. Google account is required to gain authentication token that is used to access the functions that exist in the API. Messages Scheduling Service can not be used when setting is turned off. This feature requires a storage medium and the Task Scheduler runs every minutes if the machine is connected to a wireless Internet. The whole process of data processing and feature set in a single file (engine) unless the authentication process. Quota request queries on the Google Drive API are limited: one billion requests per day, tens thousands of requests per hundred seconds, and one hundred thousand requests per second per user. These restrictions affect the system in requesting data. Parts of the system that are affected by quota restrictions are the sending new messages. This system supports the addition of another database types so in the future it can be added to achieve data exchange between different database types. The system can be further developed to support Linux operating system by using Crontab to perform task scheduling job.

\section{REFERENCES}

[1] Moh. Hidayat Koniyo, Arip Mulyanto, and Mohammad Thohir. 2014. Arsitektur Pertukaran Data Berbasis Data Grid dalam Membangun Gorontalo Library Network.

[2] Jamil Cahyadi, Marteus. U. P., and Okmonrow Muliawan. 2014. Perancangan Cloud Storage dengan Konsep Auto Syncing Menggunakan Aplikasi Owncloud dan Dropbox.

[3] I Made Sukarsa, Wisswani, and Wirabuana. "Data Exchange Between Information System at Low Bandwidth Quality Using Messaging”, JATIT, 20 February 2014 Vol. 60 No, 2.

[4] Nonprofit Technology Collaboration. 2012. Cloud Storage.

[5] Google. 2016. Clear Google Drive Space \& Increase Storage.

[6] David E. Bakken. 2003. Middleware. Washington State University, USA.

[7] I Made Sukarsa, Sarasuartha, and Arya Sasmita. "Design of Message-Oriented Middleware Based on Social Messenger”, IJCSET, April 2012, Vol. 2, Issue 4.

[8] Vahid Barekat, Ebrahim Behrouzian, and Seyed Enayattolah. "Definiton of Zachman Framework Cells Based on Service Oriented Architecture", IJSRP, 2013, Vol. 3, Issue 9.

[9] GXS. 2016. What is EDI (Electronic Data Interchange)?

[10] Data Interchange Pic. 2005. Electronic Data Interchange. Peterborough, England. 